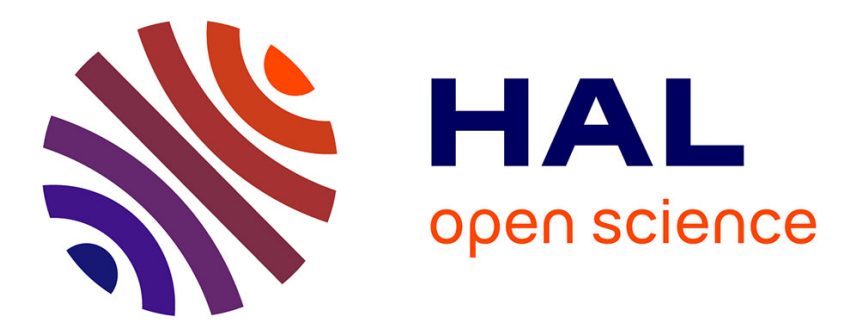

\title{
Earthquake Damage Patterns Resolve Complex Rupture Processes
}

Yann Klinger, Kurama Okubo, Amaury Vallage, Johann Champenois, Arthur

Delorme, Esteban Rougier, Zhou Lei, Earl E Knight, Antonio Munjiza, Claudio Satriano, et al.

\section{To cite this version:}

Yann Klinger, Kurama Okubo, Amaury Vallage, Johann Champenois, Arthur Delorme, et al.. Earthquake Damage Patterns Resolve Complex Rupture Processes. Geophysical Research Letters, 2018, 45 (19), pp.10,279-10,287. 10.1029/2018GL078842 . hal-02324477

\section{HAL Id: hal-02324477 \\ https://hal.science/hal-02324477}

Submitted on 16 Jul 2020

HAL is a multi-disciplinary open access archive for the deposit and dissemination of scientific research documents, whether they are published or not. The documents may come from teaching and research institutions in France or abroad, or from public or private research centers.
L'archive ouverte pluridisciplinaire HAL, est destinée au dépôt et à la diffusion de documents scientifiques de niveau recherche, publiés ou non, émanant des établissements d'enseignement et de recherche français ou étrangers, des laboratoires publics ou privés. 


\section{Geophysical Research Letters}

\section{RESEARCH LETTER}

10.1029/2018GL078842

$$
\begin{aligned}
& \text { Key Points: } \\
& \text { - Matching observed off-fault } \\
& \text { deformation pattern with rupture } \\
& \text { simulation helps identify the } \\
& \text { earthquake rupture path } \\
& \text { - Systematic consideration of off-fault } \\
& \text { damage patterns for past events } \\
& \text { would help understand earthquake } \\
& \text { scenario and to model future events } \\
& \text { This presents a great potential to } \\
& \text { narrow down the multitude of } \\
& \text { rupture scenario on complex fault } \\
& \text { system }
\end{aligned}
$$

Supporting Information:

- Supporting Information S1

- Movie S1

- Movie S2

Correspondence to:

K. Okubo,

okubo@ipgp.fr

\section{Citation:}

Klinger, Y., Okubo, K., Vallage, A., Champenois, J., Delorme, A., Rougier, E., et al. (2018). Earthquake damage patterns resolve complex rupture processes. Geophysical Research Letters, 45, 10,279-10,287. https://doi.org/ 10.1029/2018GL078842

Received 24 MAY 2018 Accepted 7 SEP 2018

Accepted article online 12 SEP 2018 Published online 2 OCT 2018

\section{Earthquake Damage Patterns Resolve Complex Rupture Processes}

\author{
Yann Klinger ${ }^{1} \mathbb{D}$, Kurama Okubo ${ }^{1,2} \mathbb{D}$, Amaury Vallage ${ }^{1,3} \mathbb{D}$, Johann Champenois ${ }^{1,4} \mathbb{D}$, \\ Arthur Delorme $^{1}$ (D), Esteban Rougier ${ }^{5}$ (D) Zhou Lei $^{5}$ (D), Earl E. Knight ${ }^{5}$ (D) Antonio Munjiza ${ }^{6}$ (D), \\ Claudio Satriano' ${ }^{1}$, Stephane Baize ${ }^{4}$ (D) Robert Langridge', and Harsha S. Bhat ${ }^{2}$ \\ ${ }^{1}$ Institut de Physique du Globe de Paris, Sorbonne Paris Cité, Université Paris Diderot, UMR 7154 CNRS, Paris, France, \\ 2Laboratoire de Géologie, École Normale Supérieure/CNRS UMR8538, PSL Research University, Paris, France, ${ }^{3}$ Now at CEA, \\ DAM, DIF, Arpajon, France, ${ }^{4}$ Seismic Hazard Division, Institut de Radioprotection et de Sûreté Nucléaire, Fontenay-aux- \\ Roses, France, ${ }^{5}$ EES-17 - Earth and Environmental Sciences Division, Los Alamos National Laboratory, Los Alamos, NM, USA, \\ ${ }^{6}$ FGAG, University of Split, Split, Croatia, ${ }^{7}$ GNS Science, Lower Hutt, New Zealand
}

Abstract Fracture damage patterns around faults induced by dynamic earthquake rupture are an invaluable record to clarify the rupture process on complex fault networks. The $2016 M_{w} 7.8$ Kaikōura earthquake in New Zealand has been reported as one of the most complex earthquakes ever documented that ruptured at least 15 crustal faults. High-resolution optical satellite image displacement maps provide distinctive profiles of displacement across the faults and help visualize the off-fault damage pattern. They are combined with field observation and coupled with a numerical tool that captures the dynamics of the rupture and simultaneous activation of off-fault damage to allow the determination of the most likely rupture scenario. This study demonstrates that complex rupture processes can be explained in a rather simple way via a synergetic combination of state-of-the-art observation and first principle physics-based numerical modeling of off-fault damage.

Plain Language Summary In a first-of-its-kind work, we combine optical image correlation at a resolution never achieved before (1.8-m ground resolution), field observations, and a unique dynamic rupture simulation tool, including off-fault deformation, to unravel, beyond doubt, part of the Kaikōura rupture process. We show that matching observed off-fault deformation pattern with rupture simulation helps easily identify the earthquake rupture path to propose a kinematically simple earthquake scenario. Thus, this work shows that when building models of seismic hazard, like in Southern California, systematic consideration of off-fault damage patterns for past events would help understand earthquake scenario and to model future events. Hence, this presents a great potential to narrow down the multitude of rupture scenarios along complex fault networks that need to be considered in seismic hazard model assessment.

\section{Introduction}

Large crustal earthquakes result from ruptures that dynamically propagate through a complex network of faults, whose temporal sequence of failure is not always clear (Hamling et al., 2017; Wei et al., 2011; Yue et al., 2012). Associated secondary faulting and coseismic off-fault damage suggest that a significant part of on- and off-fault deformation patterns is due to state of traction, fault geometry, and directivity of the rupture (Fliss et al., 2005; Kame et al., 2003; Thomas et al., 2017; Vallage et al., 2016), in addition to some geological structural inheritance (Choi et al., 2018). At ground surface, this off-fault damage zone can be hundreds of meter wide (Mitchell \& Faulkner, 2009; Vallage et al., 2015), while it becomes narrower at depth (Di Toro et al., 2005). The combined length of surface ruptures associated with the 13 November $2016 M_{w} 7.8$ Kaikōura earthquake in New Zealand (Figure 1) reaches $180 \mathrm{~km}$, distributed over more than 15 distinct fault segments (Hamling et al., 2017; Litchfield et al., 2018). Although a blind low-angle thrust might have been activated (Hollingsworth et al., 2017), the right-lateral strike-slip faults oriented NE-SW, such as the Jordan and the Kekerengu faults, dominate surface ruptures (Kääb et al., 2017; Litchfield et al., 2018; Xu et al., 2018). The 15-km-long NNW-SSE Papatea fault segment, however, is characterized by left-lateral motion of up to $\sim 6 \mathrm{~m}$ and by vertical throw reaching $10 \mathrm{~m}$ (Langridge et al., 2018). Field observations show that the northern tip of the Papatea fault does not connect to the Jordan-Kekerengu fault system (Langridge et al., 2018). All geophysical studies agree that overall the rupture propagated northward from the epicenter 


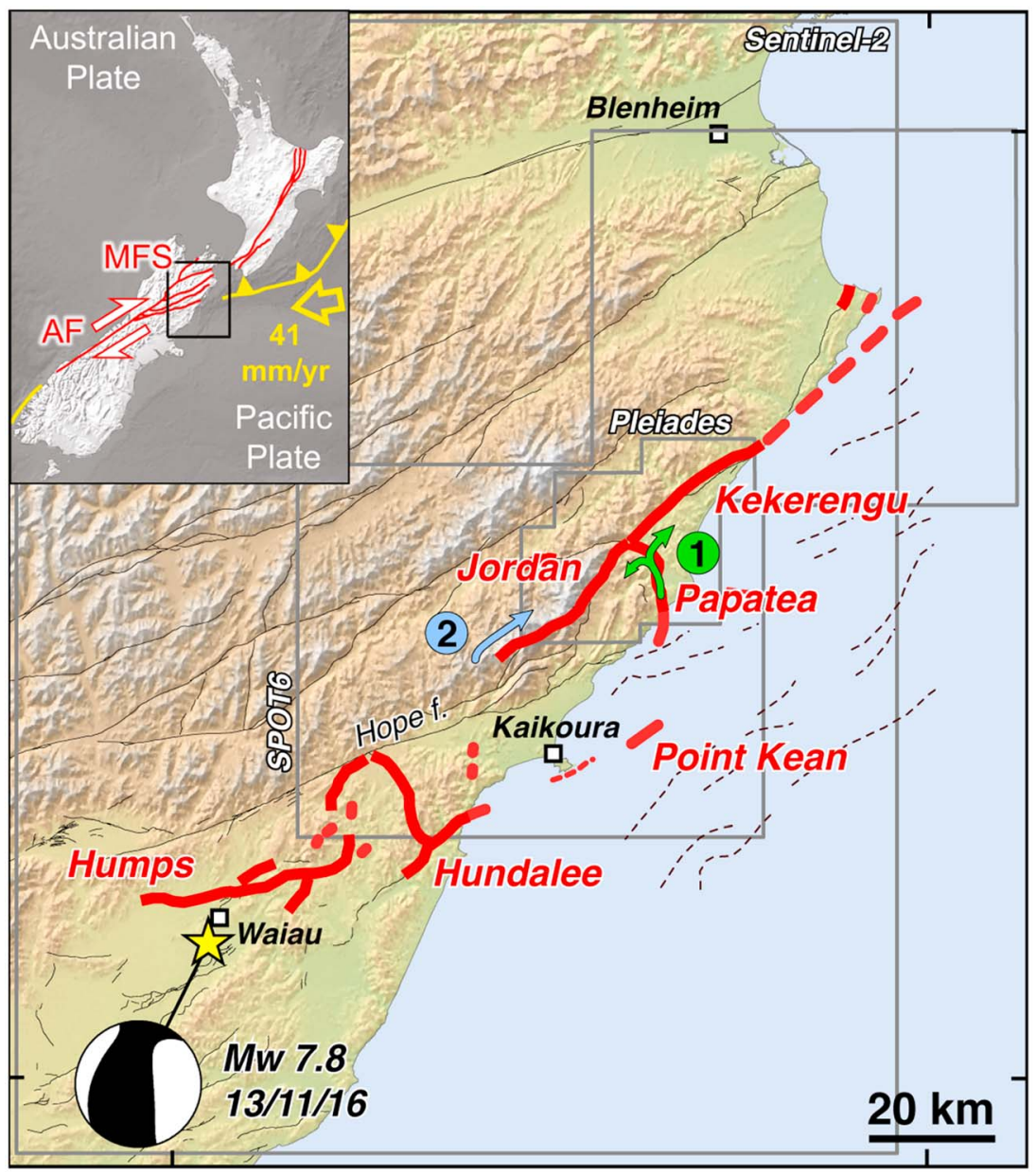

Figure 1. Map of the Kaikōura surface rupture after (Litchfield et al., 2018). Footprints for the different satellite data sets are indicated. Labels 1 and 2 refer to alternative rupture scenarios. The red solid lines indicate identified surface rupture. The Kaikoura Peninsula fault is indicated in red dashed lines. Other Quaternary faults in black. AF stands for Alpine Fault and MFS for Marlborough fault system.

(Duputel \& Rivera, 2017; Hamling et al., 2017; Wang et al., 2018; Zhang et al., 2017). However, because of the rupture complexity, partly at sea, and the possibility of a blind thrust (Hollingsworth et al., 2017), the exact rupture-propagation path remains elusive; in particular, the way the rupture propagated through the Papatea-Jordan-Kekerengu triple junction remains debated.

In this work, first we derive coseismic horizontal displacements in the triple junction area using optical satellite image correlation. The high resolution of our measurements, $1.8 \mathrm{~m}$, allows evidencing sections of the coseismic rupture with significant off-fault deformation. Then, incorporating rupture geometry derived from our measurements into dynamic rupture models that include off-fault damage, we explore alternative rupture scenarios involving the different branches of the Papatea-Jordan-Kekerengu triple junction. Eventually, comparison between optical correlation results, field observations, and our rupture simulations leads us to propose that a rupture scenario where the Papatea fault ruptured first is more likely, independently of any assumption about rupture path during earlier part of the rupture.

\section{Displacement Measurements Derived From Optical Image Correlation}

To measure the horizontal displacements associated with the 2016 Kaikōura event, we correlated optical satellite images acquired before and after the earthquake. The correlation processing was conducted by using the open-source software package MicMac (Rosu et al., 2015). The correlation was performed in two steps. In a first step, we correlated 10-m low-resolution Sentinel-2 images embracing the region where the 
Kaikōura earthquake occurred. In a second step we correlated $1.8 \mathrm{~m}$ Spot 6 images with $0.5 \mathrm{~m}$ Pleiades images to obtain a high-resolution horizontal displacement field for the Jordan-Kekerengu-Papatea triple junction.

The Sentinel-2 images were acquired respectively on 9 April 2016 and on 15 December 2016. Although the Sentinel-2 images are multispectral images with pixel resolution varying between 10 and $60 \mathrm{~m}$, here we only used the four bands with a pixel resolution of $10 \mathrm{~m}$ : red, green, blue, and near infrared. These images are orthorectified by the image provider (European Space Agency) and were correlated without any specific preprocessing. For each band, each pair of images (preearthquake and postearthquake images) was processed independently. Hence, for each component of the displacement, north-south and east-west components, we obtained four displacement maps. To improve the signal-to-noise ratio, these four maps were merged for each component of displacement, based on the median value for each quadruplet of pixels. It resulted in two horizontal-displacement maps (Figure S1), along north-south and east-west directions, which cover almost the entire Kaikōura rupture area with a ground resolution of $10 \mathrm{~m}$. The displacement maps were corrected from any low-frequency artifacts by setting the far-field displacement to zero and by crosschecking our results with Global Positioning System (https://aria-share.jpl.nasa.gov/events/20161113-NewZealand_ EQ/GPS/, last accessed July 2018) and static displacement derived from local strong motion instruments (see the supporting information). Eventually, our Sentinel-2 horizontal displacement maps compare well with previously published horizontal-displacement fields computed at similar scale from space geodesy and Global Positioning System measurements (Hamling et al., 2017; Hollingsworth et al., 2017; Kääb et al., 2017; Morishita et al., 2017; Wang et al., 2018; Xu et al., 2018).

To image details of the deformation in the close vicinity of the surface rupture, we performed correlation of metric-resolution images. For the images before the Kaikōura earthquake, we used a stereo-pair of images acquired by the satellite SPOT6 on 18 May 2014. For the images acquired after the earthquake, we used a combination of several tristereo images acquired by the satellite Pleiades between 23 December 2016 and 18 March 2017. The different subsets of Pleiades images were processed separately to ensure the best tuning of the correlation process depending on each subset, and only the final displacement maps were merged.

Images needed first to be orthorectified using a digital elevation model (DEM) with a ground resolution similar, at the first order, to the image resolution. Hence, using the Micmac package (Rosu et al., 2015), we computed preearthquake and postearthquake DEMs at the resolution $1.8 \mathrm{~m}$ from the SPOT6 images and at the resolution $0.5 \mathrm{~m}$ from the Pleiades. Because the two sets of images are from different sensors with different native resolution, the Pleiades images had to be downsampled at $1.8 \mathrm{~m}$ to be consistent with the SPOT6 images, using the open-access GDAL library (Contributors_GDAL/ORG, 2018). Eventually, a rigid translation $(\sim 10 \mathrm{~m})$ was applied to the Pleiades images, based on ground control points identified both on SPOT6 and Pleiades images, and located far from major surface ruptures, to accommodate the slight difference in absolute-location estimate between the two sensors. Then, the preearthquake and postearthquake images were correlated to compute the horizontal displacement field at the resolution of $1.8 \mathrm{~m}$. Because the area covered by high-resolution images includes almost everywhere significant coseismic deformation, zerodeformation points cannot be found to remove residual low-frequency correlation artifacts. Therefore, we used the large-scale Sentinel-2 deformation maps as a template to remove any low-frequency signal in the high-resolution displacement maps, which is not associated with tectonic deformation according to the Sentinel-2 measurements.

Eventually, using optical satellite images bracketing the date of the Kaikoura earthquake, we measured amplitude and direction of the horizontal displacement field in the triple junction area between the Jordan-Kekerengu fault system and the Papatea fault system (Figures 2 and S2). The ground resolution of our displacement field is $1.8 \mathrm{~m}$, with a displacement detection threshold of about $20 \mathrm{~cm}$ (Rosu et al., 2015). Although our measurements might include postseismic deformation, as postearthquake images were acquired between December 2016 and March 2017, it should be less than 10\% of the coseismic deformation (Hollingsworth et al., 2017) and should not affect significantly our observations.

Figure 2 shows the amplitude of the horizontal displacement at the triple junction. Systematic swath profiles every $90 \mathrm{~m}$ across different fault segments allow to establish a detailed slip distribution for that part of the Kaikōura rupture (Figures S2 and S3). Along the Jordan and the Kekerengu faults, 8-km-long swath profiles J1, J2, K1, and K2 (Figures 2, S2) show displacement parallel to the fault, where the full strike-slip 


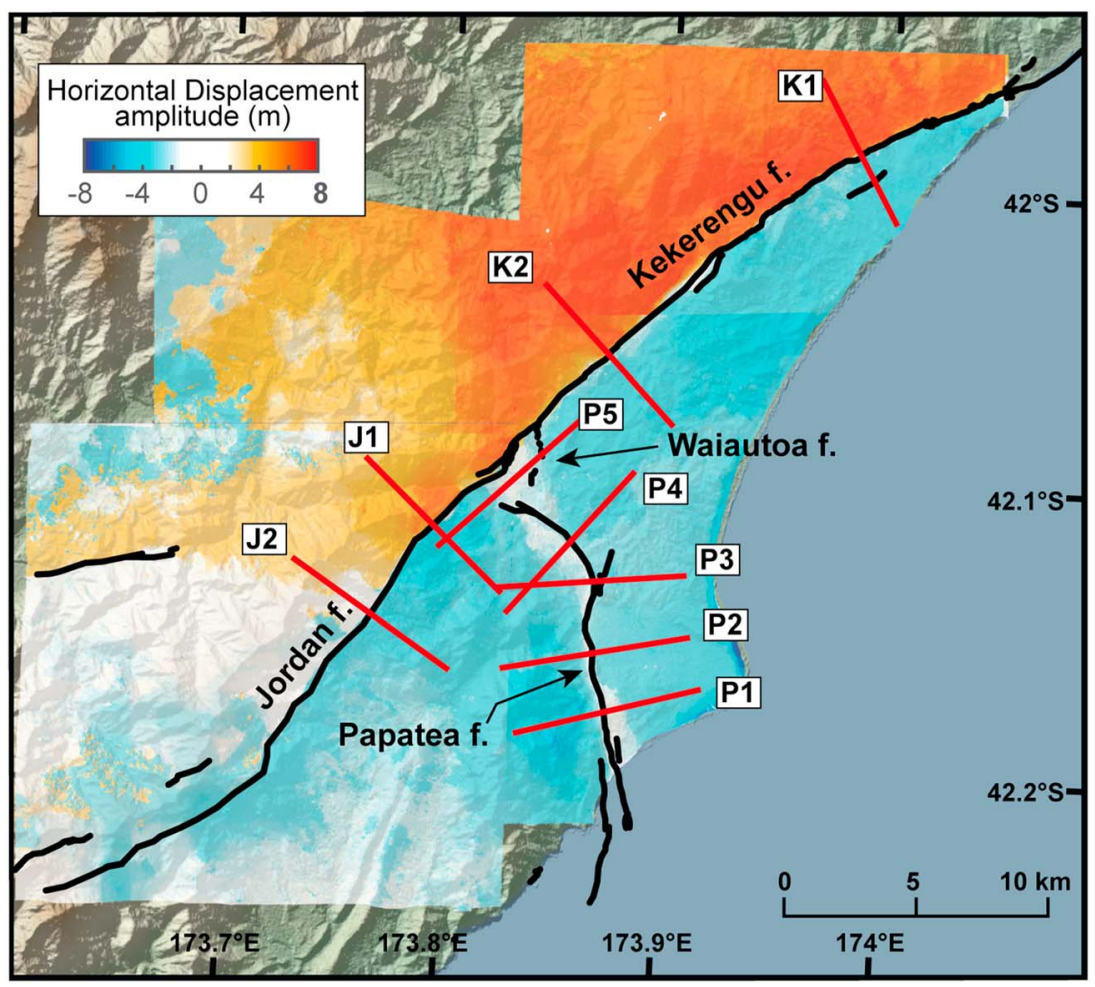

Figure 2. Displacement field around the triple junction derived from high-resolution correlation. Earthquake surface ruptures are indicated in black (Langridge et al., 2018). Color corresponds to the amplitude of horizontal displacement (positive towards north east). Low color saturation along the Papatea fault indicates off-fault damage. The red lines show the position of displacement profiles (see also Figure S1).

deformation is highly localized in a band only a few tens of meters wide. Along the Kekerengu fault, we measured a maximum right-lateral coseismic displacement of about $11 \mathrm{~m}$ (Figure S3), in good agreement with direct field-offset measurements (Kearse et al., 2018). The displacement map reveals that the pattern of deformation along the Papatea fault differs significantly from patterns along Kekerengu and Jordan faults. Along the Papatea fault, swath profiles P1 to P5 show that the gradient of horizontal deformation is not sharp everywhere (Figures 2 and S2). Instead, at both extremities, the displacement gradient is smoother, which is interpreted as distributed deformation across a damaged fault zone, as confirmed by direct field observations. Thus, the total 6-m left-lateral displacement measured along P1 is distributed over a width of $2 \mathrm{~km}$, which is consistent with field (Langridge et al., 2018) and Lidar mapping (Clark et al., 2017) that documented several parallel fault strands at the coast. Actual fault scarps in the deformation zone are indeed also visible on the profile. Along profiles P2 the deformation zone becomes narrower and asymmetric relative to the position of the fault, with most of the distributed deformation located south of Papatea. Profiles P3, P4, and P5, located north of the major bend of the Papatea fault, show that the damage zone becomes wider again, to eventually include the entire triangular zone bounded by the Papatea fault, the Kekerengu fault, and to the north-east, by the short Waiautoa fault (Figure 2).

\section{Modeling Dynamic Earthquake Rupture With Coseismic Off-Fault Damage by Continuum-Discontinuum Approach}

We used a 2-D continuum-discontinuum based scheme, the combined finite-discrete element method (FDEM), to achieve both high-numerical accuracy of rupture propagation, seismic wave radiation, and to model the activation of new cracks, in both tensile and shear, in the off-fault medium. Although vertical motion is a part of the deformation (Langridge et al., 2018; Stirling et al., 2017), at this stage we limited our study to horizontal deformation. All simulations in this work were conducted using the Hybrid Optimization Software Suite - Educational Version (HOSSedu) developed by Los Alamos National 
Laboratory. Here we only address the part of the Kaikōura rupture that concerns the Jordan-KekerenguPapatea triple junction.

Based on satellite imagery and field observations, we first defined the fault geometry in the triple junction area and discretized the domain by using an unstructured triangular mesh around the prescribed faults (Figure S4). The mesh size is adaptively controlled to be finer close to the fault to optimize trade-off between the numerical accuracy and computational cost. We then defined the initial stress state $\sigma_{\mathrm{ij}}$ uniformly in the medium. The angle of $\sigma_{1}$, the maximum principal compressive stress was set to $\mathrm{N} 107^{\circ}$, to be both compatible with the sense of slip on the different faults activated during the earthquake, in a range of $\mathrm{N} 105^{\circ}-\mathrm{N} 115^{\circ}$, and with regional focal mechanisms (Townend et al., 2012). It is assumed that the material around the faults has been previously damaged (i.e., weakened) and therefore is less competent than the rest of the material in the model. The areas of weakened material are highlighted in yellow in Figure S4a. The introduction of this weakened material area also restricted unrealistic crack propagation at the edge of faults, which could be generated due to the simple friction model (friction slip-weakening law) used here. The FDEM allows for tensile, shear, and mixed-mode crack represented as the break of cohesion at the boundary of the finite elements. In other words, each boundary of a finite element is a potential failure plane. To avoid numerical bias in the orientation of cracks, the orientations of the potential failure planes are kept isotropic (Figure S4b).

Two types of interaction forces, cohesion and contact/friction, are operating at each boundary of the finite elements, which control the failure criteria in FDEM (Figures S4c and S4d). The method is explicit in terms of time integration, so the governing equations are solved on an element-by-element basis. Evolution of cohesive and frictional forces at the interfaces, as a function of the relative displacements, is shown in Figures S4c and S4d. The opening and shear displacements, $\delta_{l}$ and $\delta_{\| \prime}$, are used to derive the cohesive forces at each time step. The first portion of the curves describing the cohesion as a function of the displacements represents a nonlinear elastic loading part, which occurs over a very small range of relative displacements between any two boundaries of finite elements. Within this range of deformation at the boundary of the finite elements, it is ensured that the entire medium behaves purely elastically since the finite element deforms as purely an elastic medium satisfying the elastic constitutive law. When the traction on the boundary of an element reaches the peak strength, then damage starts to accumulate and the cohesive strength of the interface starts to decrease linearly up to a point where it is eventually totally broken. The dissipated energy is represented by the area of triangle highlighted by slanted lines (Figures S4C and S4d). The friction curve also features an elastic loading portion, followed by the conventional linear slip-weakening law. We resolve both cohesion and friction at the interfaces of the finite elements located on the off-fault medium and only friction at the interfaces of the finite elements located along the prescribed faults; this implies that the off-fault medium is considered to be intact at the beginning of the dynamic rupture modeling. Since the fracture energy in shear is proportional to the amount of slip (Passelègue et al., 2016), the friction parameters differ between the main prescribed fault and the off-fault medium. The amount of slip is, on average, 1 or 2 orders of magnitude higher along the main fault than in the off-fault medium. When the cohesion between the finite elements starts to break, we visually plot the dynamically generated cracks, as highlighted in yellow in Figure 3a-e. The values of parameters used in our modeling are listed in Table S1. The main algorithmic solutions utilized within HOSSedu are described in (Munjiza et al., 2011).

In general, material constants, initial stress state, and frictional properties play a key role in dynamic rupture processes. We employed a homogeneous medium, which has material properties similar with granite. We then determine the peak cohesive strength for cohesion based on the closeness to failure (CF), which is defined as the ratio of the radius of the Mohr's circle to the distance to the Mohr-Coulomb criteria as shown in Figure S5a. As the material is initially intact everywhere in the medium, the CF is thus smaller than 1 across the model. We chose a CF of 0.45 , and the rest of parameters related with cohesion are derived to satisfy this condition. We then force a nucleation of the rupture by imposing a low peak strength patch around the area of nucleation. The choice of exact location of the rupture initiation is arbitrary, the goal being to ensure unilateral propagation on the targeted fault. The length of this patch is greater than the nucleation length $L_{c}$. Figure $\mathrm{S} 5 \mathrm{~b}$ shows the distribution of the initial shear traction on the prescribed fault normalized by the peak strength, $\tau^{0} / \tau^{p}$. The grid size, $d s$, along the prescribed fault is set at $50 \mathrm{~m}$. In this way, the number of finite elements in the estimated process zone size is assured to be between 8 and 14 in the entire fault system. 


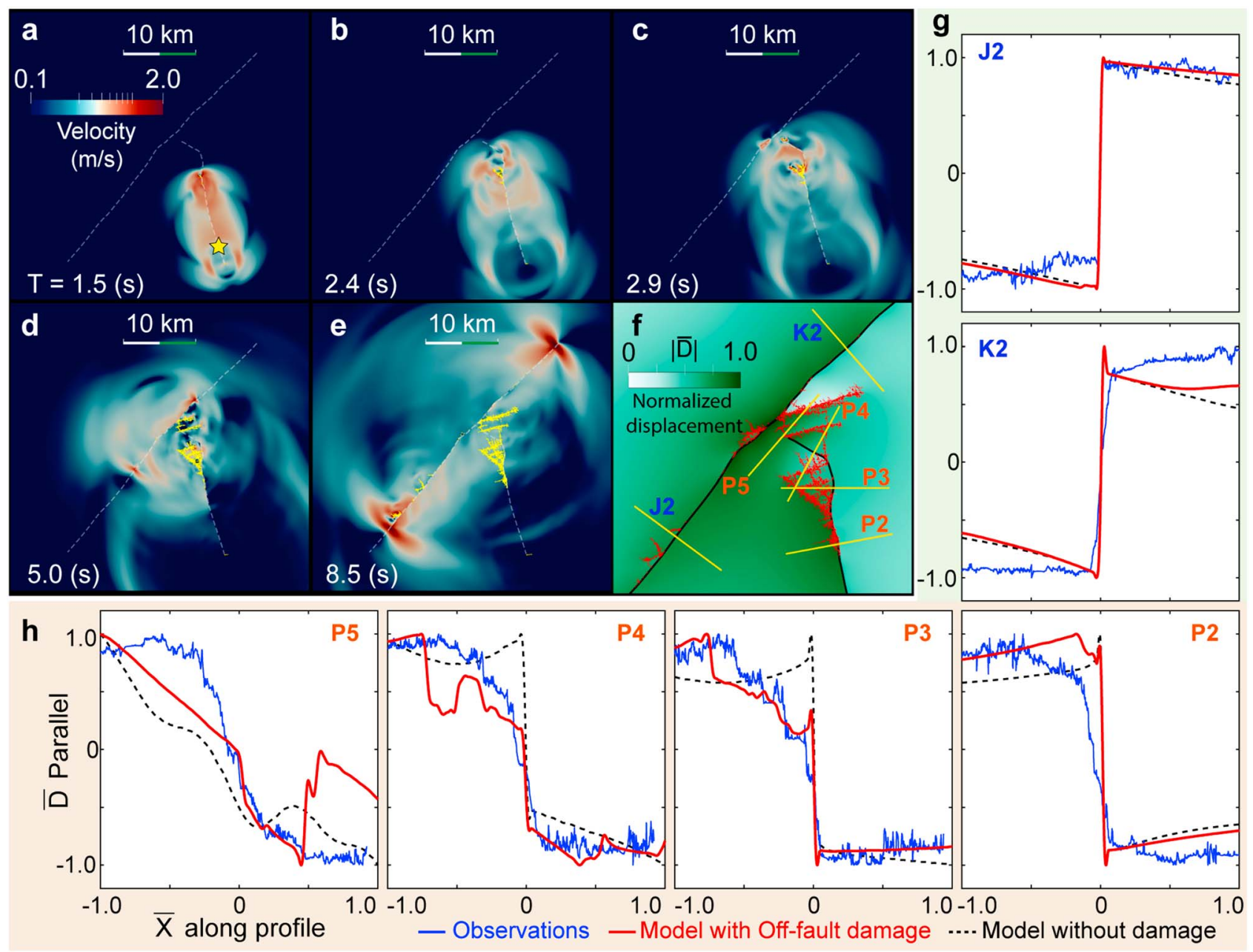

Figure 3. Rupture process, displacement field, and profiles of fault-parallel displacement for the first scenario. (a-e) Snapshots of the velocity field associated with rupture nucleated from south of Papatea fault (yellow star). The dotted lines show the prescribed faults, and the yellow lines show the spontaneously activated off-fault crack network. The color contour shows the particle velocity magnitude. (f) Deformation field and crack network at the end of the earthquake event. ( $\mathrm{g}$ and $\mathrm{h}$ ) Measured displacement (blue), modeled displacement including damage (red), and modeled displacement without damage (black dashed) for the different profiles. For comparison, both fault parallel displacements and distance along profiles are scaled by their maximum values.

\section{Earthquake Rupture Scenario and Off-Fault Damage Pattern}

Although geophysical data clearly indicate that overall the Kaikōura earthquake propagated northward, two key questions remain unsolved about the Kaikōura rupture, which are the rupture scenario of the JordanKekerengu-Papatea triple junction, and the potential activation of a shallow dipping blind thrust, possibly related to the southern end of the Hikurangi subduction (Hollingsworth et al., 2017). As for the later, our observations and modeling technics bring no additional constraints. Thus, in the following we focus only on rupture scenario for the triple junction area. Acknowledging that the rupture globally propagated northward, two classes of scenarios are possible (Figure 1), which are either a rupture first on the Papatea fault that later propagated on the Jordan-Kekerengu fault system or a rupture first on the Jordan fault that later propagated on the Kekerengu and Papatea fault systems. In the first scenario, the rupture propagated northward from the epicenter to reach the northern tip of the Hundalee fault and continued northward, offshore, until it would trigger slip on the Papatea fault. This scenario is consistent with observed coseismic uplift of the Kaikōura peninsula, which implies a shallow thrust fault outcropping offshore the Kaikōura peninsula (Clark et al., 2017), and with observation of submarine surface ruptures along the Point Kean Fault (Litchfield et al., 2018). This scenario does not rule out additional slip on a deep-seated thrust fault related to the subduction, although its amplitude would have to be limited (Clark et al., 2017). In the second scenario, when 


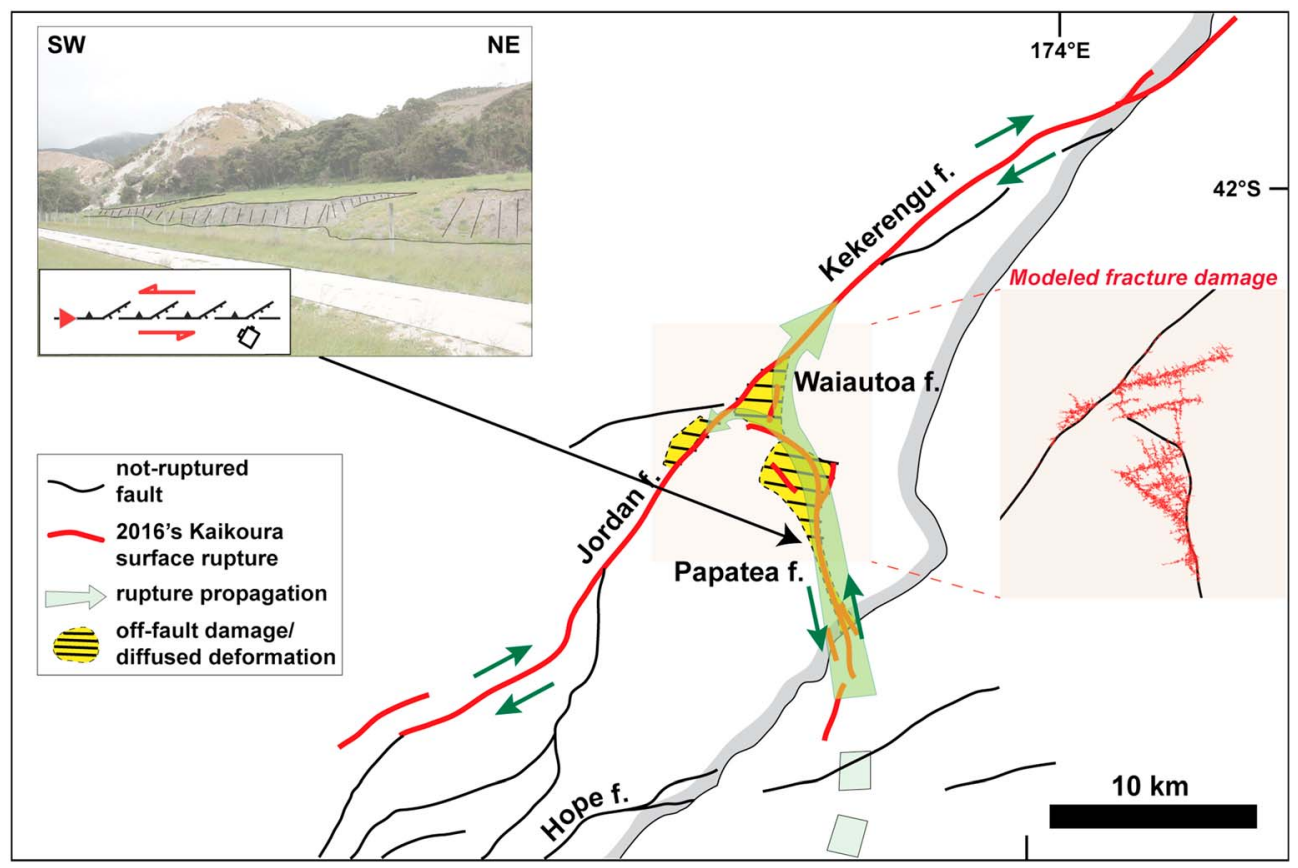

Figure 4. Summary of the preferred rupture path and associated fracture damage: Photo inset shows the observed surface damage on the Papatea fault ( $\left.542^{\circ} 08^{\prime} 47^{\prime \prime}, \mathrm{E} 173^{\circ} 52^{\prime} 01^{\prime \prime}\right)$. In addition to the main strike-slip scarp with some thrust, systematic westward branching with normal motion can be seen, which is best explained by left-lateral rupture propagating from the south. The yellow dashed areas correspond to the main areas of damage, from optical correlation and field observation.

the rupture reached the northern tip of the Hundalee fault, instead of continuing offshore the rupture would jump about $20 \mathrm{~km}$ to the NW, to dynamically trigger rupture along the Jordan fault. Limited surface ruptures were documented between the Hundalee and the Jordan faults (Litchfield et al., 2018) that might correspond to such rupture jump and potential slip along a deep-seated fault could have play some role in rupture triggering.

Here we model the two scenarios using the 2-D continuum-discontinuum model allowing for dynamic rupture propagation on prescribed faults (Figure 2) and for spontaneous activation of off-fault fracture damage. For the two scenarios, all model parameters are left identical, but for the location of the rupture initiation, which is located either along the southern part of the Papatea fault, or along the southern end of the Jordan fault. Numerical simulation of each scenario led to a distinctive pattern of rupture sequence and off-fault damage.

In the Papatea-first scenario, the rupture is initiated at the southern end of the fault and propagates northward. When it arrives at the triple junction, it triggers a new rupture on the Jordan-Kekerengu fault system, which propagates bilaterally from the junction (Figures 3a-e, MS1). While the rupture is propagating along the Papatea fault, significant damage occurs on the western side, around the main kink of the fault (Figures 3a-e). A major zone of damage also develops in the triangular zone between Kekerengu, Papatea, and Waiautoa faults. No significant damage, however, occurs along the Jordan fault (Figure 3f). Eventually, comparison of data and model outputs, both nondimensionalized, for the sake of comparison, for their spatial distribution and amplitude of displacement, shows that qualitatively they fit well (Figures $3 g, h$ ). At this stage, however, direct comparison of predicted offset values with observations, although they actually are of the same order of magnitude, is not very meaningful due to current limitation imposed by 2-D modeling whereas the Papatea fault indeed show significant vertical motion as well (Langridge et al., 2018; Stirling et al., 2017).

An alternative possibility where the Papatea rupture would have initiated north of the fault, close to the triple junction, to propagate southward and at the same time triggering rupture along the Jordan-Kekerengu fault system appears very unlikely. In fact, spatial distribution almost only west of the main rupture of off-fault 
damage identified on deformation map, as well as systematic distribution toward the NW of secondary ruptures branching off the Papatea main rupture (Figure 4), is incompatible with a southward propagation pattern (Thomas et al., 2017). In addition, rupture simulation for southward propagation shows that damage in the Papatea fault kink would make the rupture stall very quickly, impeding any further propagation southward (Figure S6).

In the Jordan-first scenario, after initiation the rupture propagates northward along the Jordan fault and eventually triggers rupture on Papatea fault. (see Figures S6c and MS2). The rupture of the Papatea fault, however, stops quickly due to significant off-fault damage (Figures S6d, e), while the rupture only continues to propagate on Kekerengu fault. Eventually, prominent damage is mostly found off Kekerengu and Jordan faults and very little off Papatea fault (Figures S6f). This is neither in agreement with the observed displacement field (Figures S6g and S6h) nor with the observed surface ruptures (Figure 4).

\section{Conclusions}

In summary, comparison of the satellite-derived displacement maps and field observation with damage patterns resulting from each modeled scenario (Figures $3 \mathrm{~g}$ and $3 \mathrm{~h}, 4$, and $\mathrm{S} 6 \mathrm{~g}$ and $\mathrm{S} 6 \mathrm{~h}$ ) shows that the Papateafirst scenario is more consistent with the observations: the Papatea fault likely ruptured from the coast toward the triple junction area where it triggered a bilateral rupture on the Jordan-Kekerengu fault system. Although it is at the current limit of the resolution of seismological data available, the seismic source studies that are focused on the second part of the Kaikōura rupture are compatible with this scenario (Holden et al., 2017; $\mathrm{Xu}$ et al., 2018) and this scenario is also fully supported by complex 3-D dynamic rupture models (Ulrich et al., 2018). More complexity, however, might still arise from full integration of vertical deformation, which is not included in our model at this stage.

Hence, although the $M_{w} 7.8$ Kaikōura earthquake has been deemed one of the most complex continental earthquake ruptures ever documented because of the very large number of fault sections activated, the general rupture mechanism might actually be simple. From the epicenter, the rupture propagated northward, navigating local geometrical complexities, then it extended offshore along the Hundalee fault and along a Kaikōura peninsula-Point Kean fault. Eventually, it dynamically triggered a rupture along the Papatea fault, located at a maximal distance of $12 \mathrm{~km}$, although it might be closer offshore. The rupture then propagated northward along Papatea and eventually triggered a bilateral rupture along the Jordan-Kekerengu fault system. The Papatea block acted as a large-scale compressional jog, which is consistent with the large documented uplift (Hamling et al., 2017; Morishita et al., 2017). If any deep-seated thrust fault was also involved, our work does not bring any additional constraints.

At first glance surface ruptures might appear very complex during large continental earthquakes, such as the Kaikōura earthquake. This complexity, however, can be resolved, and it might be that the rupture follows a rather simple structural path. Comparison between data and model outputs shows unambiguously that off-fault damage should be taken into account to explain the rupture path and the on-fault and off-fault displacement fields, which cannot be recovered by utilizing purely elastic models (dashed lines, Figures $3 \mathrm{~g}$ and 3h). Earthquake simulators used in seismic hazard assessments for complex fault systems, such as for the Southern California fault system (Field et al., 2017), can generate myriads of very large and complex fault ruptures. Providing critical keys, like off-fault damage patterns, to decipher this complexity might help narrow down a subset of most probable scenarios along complex fault networks.

The authors would like to acknowledge the constructive reviews provided by three anonymous reviewers. Numerical modeling is performed using the High Performance Computing resources provided by the Institutional Computing program at Los Alamos National Laboratory. Imagery from ESA CEOS_seismic_pilot and ISIS CNES programs processed on S-CAPAD IPGP facility. E. Rougier and H. S. Bhat are grateful to P. Johnson (LANL) for initiating a collaboration between ENS and LANL. This work was partly funded by IRSN, Campus Spatial from USPC, and CNES TOSCA programs.

\section{References}

Choi, J. H., Klinger, Y., Ferry, M., Ritz, J. F., Kurtz, R., Rizza, M., et al. (2018). Geologic inheritance and earthquake rupture processes: The 1905 $M \geq 8$ Tsetserleg-Bulnay strike-slip earthquake sequence, Mongolia. Journal of Geophysical Research: Solid Earth, 123, 1925-1953. https:// doi.org/10.1002/2017JB013962

Clark, K., Nissen, E., Howarth, J., Hamling, I., Mountjoy, J., Ries, W., et al. (2017). Highly variable coastal deformation in the 2016 Mw7. 8 Kaikōura earthquake reflects rupture complexity along a transpressional plate boundary. Earth and Planetary Science Letters, 474, $334-344$. https://doi.org/10.1016/j.epsl.2017.06.048

Contributors_GDAL/ORG (2018).GDAL/OGR geospatial data abstraction software library, edited, Open source geospatial fundation.

Di Toro, G., Nielsen, S., \& Pennacchioni, G. (2005). Earthquake rupture dynamics frozen in exhumed ancient faults. Nature, 436(7053), 1009-1012. https://doi.org/10.1038/nature03910

Duputel, Z., \& Rivera, L. (2017). Long-period analysis of the 2016 Kaikoura earthquake. Physics of the Earth and Planetary Interiors, 265, 62-66. https://doi.org/10.1016/j.pepi.2017.02.004 
Field, E. H., Jordan, T. H., Page, M. T., Milner, K. R., Shaw, B. E., Dawson, T. E., et al. (2017). A synoptic view of the third uniform California earthquake rupture forecast (UCERF3). Seismological Research Letters, 88(5), 1259-1267. https://doi.org/10.1785/0220170045

Fliss, S., Bhat, H., Dmowska, R., \& Rice, J. (2005). Fault branching and rupture directivity. Journal of Geophysical Research, 110, B06312. https:// doi.org/10.1029/2004JB003368

Hamling, I. J., Hreinsdóttir, S., Clark, K., Elliott, J., Liang, C., Fielding, E., et al. (2017). Complex multifault rupture during the 2016 Mw 7.8 Kaikōura earthquake, New Zealand. Science, 356(6334), eaam7194. https://doi.org/10.1126/science.aam7194

Holden, C., Kaneko, Y., D’Anastasio, E., Benites, R., Fry, B., \& Hamling, I. (2017). The 2016 Kaikōura earthquake revealed by kinematic source inversion and seismic wavefield simulations: Slow rupture propagation on a geometrically complex crustal fault network. Geophysical Research Letters, 44, 11,320-11,328. https://doi.org/10.1002/2017GL075301

Hollingsworth, J., Ye, L., \& Avouac, J. P. (2017). Dynamically triggered slip on a splay fault in the Mw 7.8, 2016 Kaikoura (New Zealand) earthquake. Geophysical Research Letters, 44, 3517-3525. https://doi.org/10.1002/2016GL072228

Kääb, A., Altena, B., \& Mascaro, J. (2017). Coseismic displacements of the 14 November 2016 MW7. 8 Kaikoura, New Zealand, earthquake using an optical cubesat constellation. Natural Hazards and Earth System Sciences, 17, 627-639.

Kame, N., Rice, J. R., \& Dmowska, R. (2003). Effects of prestress state and rupture velocity on dynamic fault branching. Journal of Geophysical Research, 108(B5), 2265. https://doi.org/10.1029/2002JB002189

Kearse, J., Little, T. A., Van Dissen, R. J., Barnes, P. M., Langridge, R., Mountjoy, J., et al. (2018). Onshore to offshore ground-surface and seabed rupture of the Jordan-Kekerengu-Needles fault network during the 2016 M w 7.8 Kaikōura earthquake, New Zealand. Bulletin of the Seismological Society of America, 108(3B), 1573-1595. https://doi.org/10.1785/0120170304

Langridge, R., Rowland, J., Villamor, P., Mountjoy, J., Townsend, D. B., Nissen, E., et al. (2018). Co-seismic rupture and preliminary slip estimates for the Papatea fault and its role in the 2016 Kaikoura, New Zealand, earthquake. Bulletin of the Seismological Society of America, 108(3B), 1596-1622. https://doi.org/10.1785/0120170336

Litchfield, N., Villamor, P., Van Dissen, R. J., Nicol, A., Barnes, P. M., Barrell, D. J. A., et al. (2018). Surface rupture of multiple crustal faults in the 2016 Mw 7.8 Kaiköura, New Zealand, earthquake. Bulletin of the Seismological Society of America, 108(3B), 1496-1520. https://doi.org/ $10.1785 / 0120170300$

Mitchell, T., \& Faulkner, D. (2009). The nature and origin of off-fault damage surrounding strike-slip fault zones with a wide range of displacements: A field study from the Atacama fault system, northern Chile. Journal of Structural Geology, 31(8), 802-816. https://doi.org/ 10.1016/j.jsg.2009.05.002

Morishita, Y., Kobayashi, T., Fujiwara, S., \& Yarai, H. (2017). Complex crustal deformation of the 2016 Kaikoura, New Zealand, earthquake revealed by ALOS-2. Bulletin of the Seismological Society of America, 107(6), 2676-2686. https://doi.org/10.1785/0120170143

Munjiza, A. A., Knight, E. E., \& Rougier, E. (2011). Computational mechanics of discontinua. Chichester, West Sussex: John Wiley \& Sons. https:// doi.org/10.1002/9781119971160

Passelègue, F., Schubnel, A., Nielsen, S., Bhat, H. S., Deldicque, D., \& Madariaga, R. (2016). Dynamic rupture processes inferred from laboratory microearthquakes. Journal of Geophysical Research: Solid Earth, 121, 4343-4365. https://doi.org/10.1002/2015JB012694

Rosu, A.-M., Pierrot-Deseilligny, M., Delorme, A., Binet, R., \& Klinger, Y. (2015). Measurement of ground displacement from optical satellite image correlation using the free open-source software MicMac. ISPRS Journal of Photogrammetry and Remote Sensing, 100, 48-59. https:// doi.org/10.1016/j.isprsjprs.2014.03.002

Stirling, M. W., Litchfield, N., Villamor, P., Van Dissen, R., Nicol, A., Pettinga, J., et al. (2017). The Mw 7.82016 Kaikōura earthquake: Surface fault rupture and seismic hazard context. Bulletin of the New Zealand Society for Earthquake Engineering, 2, 73-84.

Thomas, M. Y., Bhat, H. S., \& Klinger, Y. (2017). Effect of brittle off-fault damage on earthquake rupture dynamics (p. 255). Hoboken, NJ: JohnWiley.

Townend, J., Sherburn, S., Arnold, R., Boese, C., \& Woods, L. (2012). Three-dimensional variations in present-day tectonic stress along the Australia-Pacific plate boundary in New Zealand. Earth and Planetary Science Letters, 353, 47-59.

Ulrich, T., Gabriel, A.-A., Ampuero, J.-P., \& Xu, W. (2018). Dynamic viability of the 2016 Mw 7.8 Kaikōura earthquake cascade on weak crustal faults. EarthArXiv. https://doi.org/10.31223/osf.io/aed4b

Vallage, A., Klinger, Y., Grandin, R., Bhat, H. S., \& Pierrot-Deseilligny, M. (2015). Inelastic surface deformation during the 2013 Mw7.7 Balochistan, Pakistan, earthquake. Geology, 43(12), 1079-1082.

Vallage, A., Klinger, Y., Lacassin, R., Delorme, A., \& Pierrot-Deseilligny, M. (2016). Geological structures control on earthquake ruptures: The M (w)7.7, 2013, Balochistan earthquake, Pakistan. Geophysical Research Letters, 43, 10,155-10,163. https://doi.org/10.1002/2016GL070418

Wang, T., Wei, S., Shi, X., Qiu, Q., Li, L., Peng, D., et al. (2018). The 2016 Kaikōura earthquake: Simultaneous rupture of the subduction interface and overlying faults. Earth and Planetary Science Letters, 482, 44-51. https://doi.org/10.1016/j.epsl.2017.10.056

Wei, S., Fielding, E., Leprince, S., Sladen, A., Avouac, J. P., Helmberger, D., et al. (2011). Surficial simplicity of the 2010 El Mayor-Cucapah earthquake of Baja California in Mexico. Nature Geoscience, 4(9), 615-618. https://doi.org/10.1038/ngeo1213

Xu, W., Feng, G., Meng, L., Zhang, A., Ampuero, J. P., Bürgmann, R., \& et al. (2018). Transpressional rupture cascade of the 2016 Mw 7.8 Kaikoura earthquake, New Zealand. Journal of Geophysical Research: Solid Earth, 123, 2396-2409. https://doi.org/10.1002/2017JB015168

Yue, H., Lay, T., \& Koper, K. D. (2012). En échelon and orthogonal fault ruptures of the 11 April 2012 great intraplate earthquakes. Nature, 490(7419), 245-249. https://doi.org/10.1038/nature11492

Zhang, H., Koper, K. D., Pankow, K., \& Ge, Z. (2017). Imaging the 2016 MW 7.8 Kaikoura, New Zealand earthquake with teleseismic $P$ waves: A cascading rupture across multiple faults. Geophysical Research Letters, 44, 4790-4798. https://doi.org/10.1002/2017GL073461 